Meta

Journal des traducteurs

Translators' Journal

\title{
Variación terminológica: ¿Por qué y para qué?
}

\section{Judit Freixa}

Volume 50, numéro 4, décembre 2005

Pour une traductologie proactive - Actes

For a Proactive Translatology — Proceedings

Por una traductología proactiva - Actas

URI : https://id.erudit.org/iderudit/019917ar

DOI : https://doi.org/10.7202/019917ar

Aller au sommaire du numéro

Éditeur(s)

Les Presses de l'Université de Montréal

ISSN

0026-0452 (imprimé)

1492-1421 (numérique)

Découvrir la revue

Citer cet article

Freixa, J. (2005). Variación terminológica: ¿Por qué y para qué? Meta, 50(4). https://doi.org/10.7202/019917ar

\section{Résumé de l'article}

L'étude des causes de la variation terminologique a été récemment abordée (Freixa 2002), et ce qui est présenté dans cet article est une proposition méthodologique pour une analyse plus objective et profonde des causes de la variation dénominative dans les textes spécialisés.

La proposition se présente avec les résultats obtenus dans un test pilote qui a consisté dans l'entrevue directe avec les experts à propos des causes de leur propre variation terminologique. On arrive à démontrer que la typologie de classification des causes peut être modifiée et élargie à la lumière de l'analyse que les experts font de leurs propres textes, et qu'on peut parvenir à établir l’importance réelle que chacune des causes acquiert dans le discours spécialisé.
Ce document est protégé par la loi sur le droit d'auteur. L'utilisation des services d'Érudit (y compris la reproduction) est assujettie à sa politique d'utilisation que vous pouvez consulter en ligne.

https://apropos.erudit.org/fr/usagers/politique-dutilisation/ 


\title{
Variación terminológica: ¿Por qué y para qué?
}

\author{
JUDIT FREIXA \\ Universitat Pompeu Fabra, Barcelona, España \\ judit.freixa@upf.edu
}

\begin{abstract}
RÉSUMÉ
L'étude des causes de la variation terminologique a été récemment abordée (Freixa 2002), et ce qui est présenté dans cet article est une proposition méthodologique pour une analyse plus objective et profonde des causes de la variation dénominative dans les textes spécialisés.

La proposition se présente avec les résultats obtenus dans un test pilote qui a consisté dans l'entrevue directe avec les experts à propos des causes de leur propre variation terminologique. On arrive à démontrer que la typologie de classification des causes peut être modifiée et élargie à la lumière de l'analyse que les experts font de leurs propres textes, et qu'on peut parvenir à établir l'importance réelle que chacune des causes acquiert dans le discours spécialisé.
\end{abstract}

\begin{abstract}
The studies of the causes for terminological variation started not long ago (Freixa 2002). What is presented in this article is a methodological proposal for a deeper and more objective analysis of the causes for terminological variation in specialized texts.

This proposal is presented with the results obtained in a previous test. This test consisted basically in direct interviews with the experts who wrote the texts, about the causes for their own variation. The article shows that the typology of these causes can be modified and extended in the light of the analysis carried out by experts on their own texts. The test proves that it is possible to establish the real importance of every different cause in specialized discourse.
\end{abstract}

MOTS-CLÉS/KEYWORDS

terminology, term variation, specialized texts, causes of variation, experts

\section{Introducción}

Uno de los problemas terminológicos a que se enfrenta el traductor en la traducción especializada es la variación de los términos. Y es que los usos reales de los términos contradicen lo que con frecuencia se ha afirmado sobre la ausencia de variación en terminología; la teoría terminológica imperante durante décadas propone evitar la sinonimia y la polisemia para facilitar el éxito comunicativo, pero a pesar de este ideal científico, la existencia de dichos fenómenos de variación es un hecho fácilmente constatable.

Por esta razón, porque la biunivocidad de los términos no es defendible, actualmente la negación de la variación tiende a reducirse a determinadas actividades prescriptivas y, en cambio, desde un punto de vista descriptivo la variación terminológica es mayoritariamente aceptada y empiezan a existir trabajos de análisis en esta línea.

Para una buena traducción de la variación en terminología es necesario conocer las causas de la necesidad de variar y los tipos de variación resultantes. En trabajos anteriores, ya me he ocupado de un primer análisis de las causas de la variación en terminología (Freixa, 2002). Ahora me propongo ahondar en las causas estilísticas y cognitivas a partir de los elementos de soporte teórico que ofrecen los nuevos enfoques terminológicos.

\section{Primeros pasos hacia la determinación de las causas de la variación terminológica}

Como acabo de mencionar, en trabajos anteriores me ocupé de un primer análisis de las causas de la variación en terminología. En la tesis doctoral presenté una revisión de todo lo dicho anteriormente 
sobre las causas de esta variación. Para ello hice un repaso bibliográfico de los autores más relevantes que, desde las distintas corrientes de pensamiento terminológico, y tanto de forma implícita como explícita, se habían acercado a dicho tema.

A partir de toda la información recogida, presenté una tipología de las causas de la variación denominativa en los textos especializados; dicha propuesta no pretendía agotar el repertorio de causas posibles, sino más bien agrupar y articular las causas observadas. Muy brevemente, la tipología propuesta es la siguiente:

Tabla 1. Resumen de las causas de la variación denominativa (Freixa, 2002).

\begin{tabular}{|l|l|}
\hline Tipo & Subtipos \\
\hline 1. Causas previas & La redundancia lingüística \\
& La arbitrariedad del signo lingüístico \\
& Las posibilidades de variación de la lengua \\
\hline 2. Causas dialectales & Variación geográfica \\
& Variación cronológica \\
& Variación social \\
\hline 3. Causas funcionales & Adecuación al nivel de lengua \\
& Adecuación al nivel de especialización \\
\hline 4. Causas discursivas & Evitar la repetición \\
& Economía lingǘstica \\
& Creatividad, énfasis y expresividad \\
\hline 5. Causas interlingüísticas & $\begin{array}{l}\text { Convivencia del término "local" con el préstamo } \\
\text { Diversidad de propuestas alternativas }\end{array}$ \\
\hline 6. Causas cognitivas & $\begin{array}{l}\text { Imprecisión conceptual } \\
\text { Distanciación ideológica } \\
\end{array}$ \\
& Diferencias en la conceptualización \\
\hline
\end{tabular}

Finalmente, exploré la validez de la tipología de causas en un corpus de textos especializados sobre medio ambiente. La exploración me permitió constatar la pertinencia de las causas apuntadas y de la tipología propuesta. También me permitió obtener unos primeros datos acerca del peso real de las distintas causas de variación denominativa en los textos redactados por especialistas, pero no me permitió descubrir otras causas no previstas ya que no fue éste en ningún momento un objetivo del trabajo. Además, gracias a esta exploración pude establecer las limitaciones del análisis de las causas de la variación denominativa cuando se parte de textos ya producidos.

\section{Nuevos pasos hacia hacia la determinación de las causas de la variación terminológica}

Sin pretender invalidar el trabajo realizado, ya que lo entiendo como un primer paso imprescindible, considero que analizar el texto especializado ya producido e hipotetizar las causas de la aparición de variación denominativa es no solamente insuficiente, sino necesariamente superable ${ }^{1}$.

\section{Nuevas posibilidades metodológicas}

Sería óptimo poder intervenir en el proceso de producción de un texto cuando lo lleva a cabo el especialista, pero este tipo de estudio no parece fácilmente realizable, más allá del análisis de las versiones del texto hasta llegar al texto definitivo, análisis que también presentaría limitaciones importantes.

Otra posibilidad de superación metodológica sería, con un enfoque cognitivo, realizar encuestas a especialistas sobre la recepción de la variación denominativa que ofrece un texto especializado, aunque de nuevo se estaría hipotetizando sobre algo que ha escrito un especialista; la mejora, en este caso, sería que los sujetos interpretativos serían semejantes al productor del texto, y 
no investigadores externos a la temática y a los textos, como sucede cuando el análisis lo realiza el lingüista.

Sin ninguna duda, la opción más económica, en el sentido de más fácilmente realizable y que más información fiable puede aportar, es realizar encuestas a los propios productores de los textos y sobre textos ya producidos. Interrogar directamente al especialista sobre la causa de sus propias variaciones.

\section{Nuevos supuestos de partida e hipótesis}

Para este trabajo parto de dos supuestos fundamentales: el primero, que no es lo mismo la variación en un mismo autor (autovariación) que la variación entre autores distintos (heterovariación), y el segundo, que no es lo mismo la autovariación en un mismo texto que la autovariación entre textos diferentes. La hipótesis que subyace en ambos supuestos es que las causas no son idénticas entre autovariación $(=\mathrm{A}$, mismo autor) y heterovariación $(\neq \mathrm{A})$, ni dentro de un mismo texto $(=\mathrm{T}) \mathrm{o}$ textos distintos $(\neq \mathrm{T})$ escritos por un mismo autor. La relación que establezco es la siguiente:

- causas dialectales: $\neq \mathrm{A}$;

- causas funcionales: $=\mathrm{A} \neq \mathrm{T}$;

- causas discursivas: $=\mathrm{A}=\mathrm{T}$;

- causas interlingüísticas: $=\mathrm{A}$ o $\neq \mathrm{A}$;

- causas cognitivas: $=$ A o $\neq$ A (dependiendo del subtipo de causa cognitiva).

Otra hipótesis de este trabajo es que el especialista no realiza todas las variaciones denominativas con el mismo nivel de conciencia; algunas variaciones son conscientes y deseadas, y persiguen un objetivo definido, pero otras son inconscientes y la finalidad (si la hay) se encuentra en un nivel de definición diferente.

\section{Nuevos objetivos e interrogantes}

En este trabajo pretendo centrarme solamente en la variación de un mismo autor en un mismo texto $(=\mathrm{A}=\mathrm{T}) \mathrm{y}$, por lo tanto, dejo a un lado las causas dialectales (que provoca directamente $\neq \mathrm{A}) \mathrm{y}$ funcionales (que provoca directamente $\neq \mathrm{T}$ ).

Pretendo también comprovar las hipótesis, es decir, ver si la relación hipotetizada entre la situación de variación y las causas es cierta, y ver como se concreta esta relación. Me interesa comprobar si la entrevista directa con los especialistas puede aportar matices importantes a las causas previstas para la variación terminológica.

Por otro lado, es también un objetivo del trabajo avanzar en el conocimiento sobre qué variaciones son conscientes y cuales son inconscientes; cual es el objetivo perseguido y por qué se persigue, especialmente cuando las variaciones son conscientes.

En el fondo, me interesa establecer por qué y para qué un autor varía, es decir, por qué el ideal de la biunivocidad pasa a un segundo nivel de prioridad. ¿Qué hay más importante que asegurar la fórmula de "un concepto para cada denominación, y una denominación para cada concepto":

- ¿Se trata tal vez de una cuestión estética porque prima la belleza discursiva?

- ¿o es una cuestión más profunda, de carácter cognitivo?

- ¿o es una razón más práctica, relativa al contacto entre lenguas?

¿Y la eficacia comunicativa? ¿Por qué siempre se ha asociado la eficacia comunicativa con la biunivocidad? ¿Tal vez para ser eficaces tenemos que variar, contrariamente a lo que siempre se había creído (en relación a los textos especializados)? ¿O simplemente es la explosión de los escenarios comunicativos (más tipos de emisores, más tipos de receptores, más tipos de textos, más canales de comunicación, etc.) lo que ha provocado un nuevo concepto de eficacia comunicativa? 


\section{La propuesta de investigación}

Para superar las limitaciones metodológicas manifestadas más arriba y para, a partir de los supuestos de partida e hipótesis, dar respuesta a los interrogantes, me propongo realizar una investigación basada en entrevistas directas a especialistas sobre las causas de la variación denominativa presente en los textos redactados por ellos mismos.

Desde un punto de vista metodológico, esta investigación se realizará de acuerdo con las siguientes fases:

a) creación de un corpus textual;

b) selección de los textos y de los fragmentos;

c) preparación de los textos y de la entrevista;

d) obtención de los datos (grabación de la entrevista);

e) análisis de los resultados.

\section{Prueba piloto}

Lo que voy a presentar a continuación es una prueba piloto realizada para evaluar la viabilidad y el interés de la investigación propuesta. En primer lugar voy a presentar una descripción de la prueba y, a continuación, los resultados obtenidos. Para ello voy a seguir las cinco fases presentadas más arriba.

\section{a) Creación de un corpus textual}

Para la investigación pienso trabajar con textos de diferentes áreas de especialidad, pero para la prueba piloto me he centrado en textos del área de linguiística; concretamente, textos sobre teoría terminológica redactados por miembros de nuestro grupo de investigación (Grupo IULATerm); textos redactados en castellano y publicados (o en curso de publicación) en los últimos años en monografías especializadas ${ }^{2}$.

Las razones de dicha elección resultan más que obvias: fácil acceso a los textos; conocimiento profundo del tema (lo cual facilita la detección de la variación); fácil acceso a los redactores de los textos (fácil obtención de entrevistas); fácil comprensión del experimento por parte de los entrevistados.

Los sesgos de mi elección también resultan bastante obvios y hay que tenerlos en cuenta en la interpretación y generalización de los resultados:

- en relación a los textos: tal vez ni la cantidad de variación que aparece en los textos especializados sobre terminología, ni los tipos predominantes, son extrapolables a textos especializados de otras temáticas;

- en relación a los especialistas: la capacidad metalingüística de los lingüistas no es extrapolable a los demás colectivos especializados; y todavía menos extrapolable es la capacidad interpretativa de sus variaciones que tienen los expertos en terminología ubicados en un marco de investigación donde la variación lingüística es un fenómeno muy analizado y conocido.

\section{b) Selección de los textos y de los fragmentos}

Del conjunto de textos disponibles, he escogido aquellos que están redactados por un solo especialista (para asegurarme que la encuesta sea ciertamente sobre la variación de uno mismo) y que presentan variación. Lo que me interesa es descubrir porqué se varía cuando se varía y no si se varía o no ${ }^{3}$. Así pues, he seleccionado aquellos textos (solamente la parte inicial, las primeras páginas) en los que me ha resultado fácil detectar conceptos con alta frecuencia de aparición y denominados de formas distintas. 


\section{c) Preparación de los textos y de la entrevista}

Sobre la versión electrónica de los textos, la preparación para la entrevista ha consistido, en primer lugar, en la detección de las distintas denominaciones (u otras formas) para un mismo concepto. Así, he seleccionado aquellas ocurrencias en las que el concepto aparecía bajo una forma denominativa y he descartado los ejemplos de variación formal no denominativa ${ }^{4}$. Por ejemplo, en el texto 2 he recogido, para el concepto "fraseología especializada", denominaciones como "combinaciones fraseológicas especializadas" o "combinaciones terminológicas recurrentes" y se han dejado de lado formas como "este tipo de combinaciones léxicas" o "estas expresiones".

La preparación de los textos ha consistido además en el marcaje de la primera ocurrencia con la primera denominación (denominación que ha recibido el nombre de "denominación de referencia") y, finalmente, en la substitución del resto de denominaciones por espacios en blanco (siempre de la misma longitud) para que el entrevistado escriba la denominación correspondiente.

Vemos la transformación del texto en el ejemplo siguiente; en primer lugar se presenta el fragmento original con las denominaciones detectadas (subrayado) y, en segundo lugar, el fragmento preparado con la supresión de denominaciones (excepto en la primera ocurrencia):

\subsection{Las UT monoléxicas simples}

Las UT monoléxicas simples son difíciles de tratar automáticamente porque su carácter especializado es totalmente idiosincrático. Son, pues, un tipo de unidades que no poseen características, ni morfológicas, ni sintácticas explícitas, que permitan detectarlas automáticamente. [...] El análisis de corpus lexicográficos y textuales demuestra que el número de términos simples en relación con otras unidades especializadas es muy bajo. No obstante, es interesante poder extraer de los textos las UT simples porque son una pieza clave del léxico especializado [...].

\subsection{Las UT monoléxicas simples}

Las _________ son difíciles de tratar automáticamente porque su carácter especializado es totalmente idiosincrático. Son, pues, un tipo de unidades que no poseen características, ni morfológicas, ni sintácticas explícitas, que permitan detectarlas automáticamente. [...] El análisis de corpus lexicográficos y textuales demuestra que el número de en relación con otras unidades

especializadas es muy bajo. No obstante, es interesante poder extraer de los textos las porque son una pieza clave del léxico especializado [...].

\section{d) Obtención de los datos (grabación de la entrevista)}

La entrevista empieza mostrándole al especialista entrevistado el texto que se analiza. Una vez lo ha reconocido, se le muestra el fragmento escogido y se le informa del concepto (o de los conceptos) que constituyen el objeto del análisis. El especialista sabe que se trata del análisis de su propia variación denominativa.

A continuación se le ofrece el texto con los segmentos vacíos y se le pide que los rellene. Una vez ha terminado, empieza la grabación de la conversación en la cual se le comenta caso por caso si ha coincidido o no con la versión original del texto. Las preguntas directas al entrevistado dependen en cada caso de la situación en relación a la variación:

en el texto original no había variación y ahora sí la hay;

en el texto original no había variación y ahora tampoco la hay;

en el texto original había variación y ahora no la hay;

en el texto original había variación y ahora también la hay, pero es distinta;

en el texto original había variación y ahora también la hay, y es idéntica.

Las preguntas (del tipo “¿antes no habías variado, por qué ahora sí?”, “¿por qué no has utilizado la denominación de referencia?”, “¿por qué introduces esta nueva denominación?”, “¿significa realmente lo mismo?”, etc.) también dependen de si el propio especialista va comentando y argumentando su variación o si no lo hace. 
Al final del comentario se le presenta un resumen de la variación detectada en el texto original, se comentan todas las denominaciones utilizadas para el mismo concepto y se le pregunta si todas estas formas son realmente denominaciones, si alguna o algunas de ellas está más fijada que las otras, y el papel que realizan las formas alternativas.

\section{e) Análisis de los resultados}

Aunque para el análisis se han utilizado todos los resultados, en esta presentación se ofrecen sólo algunos ejemplos. El análisis se presenta de acuerdo con los tres bloques de resultados siguientes: i) La variación en los textos analizados, donde se analiza la variación denominativa detectada en los textos originales; ii) ¿Variación consciente o inconsciente?, donde se presenta el contraste con los resultados de variación producidos por los especialistas en los textos modificados; y iii) El especialista opina, donde se analizan las conversaciones grabadas sobre las causas de la variación.

\section{Primer bloque: La variación en los textos analizados}

Como ya se ha dicho, en la prueba piloto se ha trabajado con tres textos, y se ha analizado la variación que presentan los seis conceptos siguientes:

T1: unidades terminológicas (C1), UT monoléxicas simples (C2) y UT derivadas (C3)

T2: fraseología especializada (C4) y aplicaciones terminográficas (C5)

T3: grupos profesionales (C6)

La tabla siguiente muestra un resumen de los resultados obtenidos. En la primera columna se identifica el concepto analizado con el número atribuido más arriba; en la segunda columna se ofrece la denominación de referencia (el número que aparece entre paréntesis tras cada denominación se refiere al número de ocurrencias de esa denominación); en la tercera columna se presentan las denominaciones alternativas documentadas para el mismo concepto (y también se indica su frecuencia); finalmente, en la cuarta columna se ofrece el índice de denominación ${ }^{5}$ de dicho concepto, calculado a partir de la división del número de denominaciones por el número de ocurrencias.

Tabla 2. La variación en los textos originales.

\begin{tabular}{|c|c|c|c|}
\hline Núm. & Denominación de referencia & Denominaciones alternativas & Índice \\
\hline $\mathrm{C} 1$ & unidades terminológicas (1) & $\begin{array}{l}\text { UT (9) } \\
\text { USE (2) } \\
\text { unidades léxicas de significado especializado } \\
\text { (1) } \\
\text { unidad de significación especializada (1) } \\
\text { unidades (1) }\end{array}$ & $\begin{array}{l}6 \text { den } / 15 \text { oc } \\
=\mathbf{0 , 4}\end{array}$ \\
\hline $\mathrm{C} 2$ & UT monoléxicas simples (1) & $\begin{array}{l}\text { términos simples (1) } \\
\text { UT simples (1) } \\
\text { USE simples (3) } \\
\text { unidad de significación simple (1) } \\
\text { USE monoléxicas simples (1) }\end{array}$ & $\begin{array}{l}6 \text { den } / 8 \text { oc } \\
=\mathbf{0 , 7 5}\end{array}$ \\
\hline $\mathrm{C} 3$ & UT derivadas (1) & $\begin{array}{l}\text { USE derivadas (1) } \\
\text { USE monoléxicas derivadas (1) }\end{array}$ & $\begin{array}{l}3 \mathrm{den} / 3 \mathrm{oc} \\
=\mathbf{1}\end{array}$ \\
\hline $\mathrm{C} 4$ & fraseología especializada (4) & $\begin{array}{l}\text { fraseología (3) } \\
\text { combinaciones terminológicas recurrentes (1) } \\
\text { fenómenos fraseológicos (1) } \\
\text { combinaciones fraseológicas especializadas } \\
\text { (1) } \\
\text { unidades fraseológicas (2) }\end{array}$ & $\begin{array}{l}6 \text { den } / 12 \text { oc } \\
=\mathbf{0 , 5}\end{array}$ \\
\hline
\end{tabular}




\begin{tabular}{|l|l|l|l|}
\hline C5 & aplicaciones terminográficas (2) & $\begin{array}{l}\text { vocabularios especializados (2) } \\
\text { recursos (1) } \\
\text { diccionarios (1) } \\
\text { aplicaciones (1) } \\
\text { actividad terminológica (1) }\end{array}$ & $\begin{array}{l}6 \text { den } / 8 \text { oc } \\
\mathbf{0 , 7 5}\end{array}$ \\
\hline C6 & grupos profesionales (6) & $\begin{array}{l}\text { grupos (15) } \\
\text { grupos especializados (5) } \\
\text { grupos de especialistas(1) } \\
\text { grupos sociales (1) } \\
\text { grupos sociales especializados (1) } \\
\text { grupos sociales profesionales (2) }\end{array}$ & $\begin{array}{l}7 \text { den } / 31 \text { oc } \\
\mathbf{0 , 2 2}\end{array}$ \\
\hline
\end{tabular}

A la vista de los resultados de la tabla se pueden destacar al menos los aspectos siguientes:

- el grado de variación es alto: los conceptos analizados están representados por un número elevado de denominaciones; solamente en C3 se han obtenido menos de cuatro denominaciones pero justamente se trata del caso más espectacular: encontramos tres denominaciones distintas para las tres únicas ocurrencias. O lo que es lo mismo: en cada ocasión el concepto ha sido denominado de una forma distinta;

- el grado de variación parece todavía mayor si tenemos en cuenta la frecuencia de aparición, es decir, si nos fijamos en el índice de denominación: dado que, como ya he mencionado, dicho índice se obtiene dividiendo el número de denominaciones por el número de ocurrencias, el índice máximo és 1 (lo que supone una denominación distinta para cada ocurrencia) y el mínimo sería la cifra más cercana a 0 (sin poder llegar nunca a 0 ). En los resultados vemos que este índice varía entre los dos extremos: en el último caso (C6) es bajo $(0,22)$ y en el tercero (C3) és el máximo de alto (1); en los demás casos es bastante elevado $(\mathrm{C} 1=0,4, \mathrm{C} 4=0,5)$ o muy elevado $(\mathrm{C} 2$ y $\mathrm{C} 5=0,75)$;

- aunque no sea uno de los objetivos del trabajo, es importante mencionar que el nivel de equivalencia conceptual (EC) de las unidades en variación es también muy heterogéneo: muy elevado cuando la variación se produce entre un término desarrollado y su sigla, bastante elevado en casos de reducciones anafóricas, y mucho más bajo en otros ejemplos que analizaremos más adelante.

\section{Segundo bloque: ¿variación consciente o inconsciente?}

Como he dicho anteriormente, un objetivo del trabajo es avanzar en el conocimiento sobre qué variaciones son conscientes y cuáles son inconscientes, aspecto que paso a discutir en este segundo bloque de resultados.

El especialista actúa libremente cuando redacta su texto original, e introduce variación consciente o inconscientemente, pero parto de la base de que por el simple hecho de hacerle reescribir el texto y de interrogarle sobre sus cambios denominativos, la espontaneidad decrece considerablemente y, por tanto, aumenta el grado de conciencia sobre la variación.

Teniendo en cuenta el sesgo que introduce esta limitación, considero que el especialista debería repetir los usos denominativos (variados o no) conscientes y que difícilmente habrá coincidencia en aquellos usos más inconscientes. Así pues, para determinar el grado de conciencia me apoyo en el grado de coincidencia, aunque después me apoyo también en la interrogación directa al entrevistado.

En la tabla siguiente se ofrecen los resultados numéricos en relación a la coincidencia o no coincidencia de los usos denominativos por parte del especialista entre el texto original y el texto rellenado durante la entrevista. 
Tabla 3. Grado de coincidencia denominativa.

\begin{tabular}{|l|l|l|l|}
\hline Núm. & Ocurrencias & Coincidentes & No coincidentes \\
\hline C1 & 15 & $9(60 \%)$ & $6(40 \%)$ \\
\hline C2 & 8 & $1(12,5 \%)$ & $7(87,5 \%)$ \\
\hline C3 & 3 & $1(33,3 \%)$ & $2(66,6 \%)$ \\
\hline C4 & 12 & $7(58,3 \%)$ & $5(41,6 \%)$ \\
\hline C5 & 8 & $1(12,5 \%)$ & $7(87,5 \%)$ \\
\hline C6 & 31 & $17(54,8 \%)$ & $14(45,1 \%)$ \\
\hline
\end{tabular}

También en esta ocasión, los resultados son dispares: una coincidencia del 60\% (o del 58\%) se podría considerar muy elevada, y una coincidencia del $12,5 \%$, se consideraría decididamente baja. Claro está que en este caso la información numérica es muy opaca, y parece más relevante la información cualitativa: ¿qué tipo de coincidencias hay? y ¿qué tipo de no coincidencias?

Para no sobrecargar la presentación con datos y ejemplos, vamos a analizar solamente lo sucedido en un ejemplo de elevada coincidencia (C1) y en un ejemplo de baja coincidencia (C5) (como ya se ha dicho, en el anexo se pueden consultar todos los resultados obtenidos):

Tabla 4. Análisis de la coincidencia denominativa en C1.

\begin{tabular}{|l|l|}
\hline Texto original & Texto entrevista \\
\hline unidades terminológicas (1) & unidades terminológicas (3) \\
\hline UT (9) & UT (12) \\
\hline USE (2) & \\
\hline unidades léxicas de significado especializado (1) & \\
\hline unidad de significación especializada (1) & \\
\hline unidades (1) & \\
\hline
\end{tabular}

Como se puede ver, el índice de variación ha disminuido muy considerablemente en este ejemplo (de 6 denominaciones hemos pasado a 2). El especialista ha utilizado la denominación de referencia en tres ocasiones ( $y$ no solamente en una, como en el texto original) y en el resto de ocurrencias ha recurrido a la sigla directa de la denominación de referencia. En el texto de la entrevista no se ha recurrido en ningún momento al uso de otras formas alternativas, más o menos equivalentes utilizadas en el texto original (como "unidades léxicas de significado especializado" o "unidad de significación especializada").

Los resultados de este primer ejemplo ya nos permite afirmar que no toda la variación denominativa es consciente. Sí lo es el uso de la forma abreviada (UT) por la forma desarrollada ("unidades terminológicas"), pero no lo son las demás formas que permiten variar la expresión aunque a nivel conceptual no haya equivalencia absoluta: el contexto impide que esta variación menos exacta resulte ambigua o ineficaz.

En cualquier caso, la comparación de la variación denominativa presente en el texto original con la de la variación en el texto preparado para la entrevista permite afirmar que la decisión de variar no era muy consciente en este primer ejemplo.

Tabla 5. Análisis de la coincidencia denominativa en C5.

\begin{tabular}{|l|l|}
\hline Texto original & Texto entrevista \\
\hline aplicaciones terminográficas (2) & aplicaciones terminográficas (1) \\
\hline diccionarios (1) & diccionarios (1) \\
\hline recursos (1) & \\
\hline vocabularios especializados (2) & \\
\hline aplicaciones (1) & \\
\hline actividad terminológica (1) & \\
\hline
\end{tabular}




\begin{tabular}{|l|l|}
\hline & vocabularios (2) \\
\hline & vocabularios o BD (1) \\
\hline & vocabularios terminológicos (1) \\
\hline & aplicaciones terminológicas (1) \\
\hline & terminografía (1) \\
\hline
\end{tabular}

Resulta sorprendente el bajísimo grado de coincidencia que presenta la variación denominativa en este ejemplo: en el texto original encontramos 6 denominaciones diferentes en solamente 8 ocurrencias distintas; pero en el texto de la entrevista, el número de denominaciones es todavía mayor ( 77 denominaciones!). Lo que resulta más sorprendente es que de estas 6/7 denominaciones para un mismo concepto solamente coinciden 2 , lo que eleva a 11 el número total de denominaciones.

Este ejemplo permite afirmar que existe una clara consciencia de la necesidad de variar y, en cambio, una gran libertad para el tipo de salida que se da a esta necesidad lingüística. Así, en el ejemplo vemos el uso indistinto de formas con un valor conceptual diferente para hacer referencia a un mismo concepto; son, pues, denominaciones alternativas con un grado de equivalencia conceptual bajo respecto de la denominación de referencia.

\section{Tercer bloque: el especialista opina.}

Como ya se ha dicho, una vez el especialista había rellenado los espacios vacíos (para constatar si variaba del mismo modo o de modo distinto), se llevó a cabo una grabación de la conversación en la que se contrastaban sus usos denominativos en el texto original con los del nuevo. Intenté que el propio especialista opinara libremente al darse cuenta de cuánto había variado en el texto original, de las diferencias entre los dos textos, etc. Pero no dudé tampoco en hacer preguntas directas sobre las razones, las causas, la validez de las denominaciones, el grado de equivalencia conceptual, etc.

De todo lo comentado en las tres entrevistas voy a referirme al menos a los 4 aspectos siguientes:

\section{i) primacía del factor estilístico}

Como era de esperar, todos los especialistas dieron como causa fundamental (o única) de sus variaciones el interés por crear un texto estilísticamente aceptable; así, se usó la variación (es decir, formas denominativas alternativas a la denominación de referencia) para evitar ser redundante ["digo 'vocabularios' en vez de 'vocabularios especializados' porque se entiende por el contexto y no quiero resultar tan redundante"]. En realidad, tras esta razón se encuentran dos razones más concretas, también expresadas por los especialistas entrevistados: evitar ser repetitivo e intentar ser económico.

Para ello, como ya hemos visto anteriormente en los ejemplos, los especialistas no dudan en realizar cualquier tipo de reducción anafórica contextual (que justifican diciendo que de este modo el significado no se ve en absoluto alterado y la forma sólo parcialmente) o en crear siglas (algunos especialistas son mucho más propensos a la creación in situ de siglas que otros). Y cuando lo prioritario es variar (y no la economía lingüística), los especialistas utilizan formas más descriptivas y parafrásticas, formas menos lexicalizadas que permiten reconocer el concepto sin ambigüedades.

Lo que subyace en esta necesidad es, tal como lo expresa uno de los especialistas "aligerar un poco el texto, que bastante pesado resulta".

\section{ii) menoscabo del factor cognitivo}

$\mathrm{Al}$ inicio del trabajo ya he manifestado que no se iban a analizar las causas dialectales ni funcionales de la variación denominativa, dado que me he centrado en la variación de un mismo autor dentro de un mismo texto. La posibilidad de aparición de la causa interlingüística era existente, pero no se ha dado. Así pues, más allá de las causas estilísticas o discursivas, el factor cognitivo es el que, realmente, podría haber tenido un peso importante. Y no ha sido así, contrariamente a mis hipótesis. 
Los especialistas no se han referido de forma espontánea en ningún momento a una supuesta causa cognitiva y, cuando yo la he planteado, la han negado de forma sistemática.

Plantée la pregunta "podría ser que en este caso estuvieses enfocando el concepto desde una perspectiva concreta, que estuvieses haciendo énfasis en la parte x del concepto" a cada uno de los especialistas en los casos siguentes:

- en el texto 1, para la alternancia entre "fraseología" y "combinatoria";

- en el texto 2 para la alternancia entre "USE derivadas" y "UT derivadas" y entre "USE monoléxicas derivadas" y "UT derivadas";

- y en el texto 3, para la alternancia entre "grupo social" y "grupo profesional" y también entre "grupos sociales especializados" y "grupos profesionales".

Y la respuesta obtenida fue siempre la negación de mi propuesta de forma casi categórica; solamente en un caso obtuve un escéptico "no sé".

Podría, por supuesto, considerar que todos ellos se equivocan y que no reconocen la causa de sus propias variaciones y que en cambio, yo sí la reconozco, pero me resulta incómodo afirmar algo así: se trata de colegas que son expertos lingüistas... Aunque también me resulta incómodo aceptar simplemente que erré la hipótesis, y todavía más cuando me fijo en el tipo de variaciones denominativas (tan distintas a las vistas en el apartado de causas estilísticas) que se producen en todos estos ejemplos: auténticas variaciones léxicas que cambian (más que añaden o quitan) rasgos semánticos del concepto explicitados en la denominación.

Me fijo como objetivo prioritario para mi investigación encontrar una explicación razonable para esta situación, ya que en este momento los datos obtenidos no me la ofrecen. Podría ser que el factor cognitivo opere en un nivel tan profundo (inconsciente) que el especialista no pueda reconocerlo en una entrevista tan corta y rápida. Un elemento a favor de este razonamiento sería el hecho de que en los ejemplos explicados por el factor estilístico, los especialistas han coincidido de forma muy elevada en los usos denominativos de los dos textos (texto original y texto modificado), lo que me ha llevado a considerarlos conscientes. Y, en cambio, no se daba tal coincidencia entre los dos textos para los ejemplos que yo considero de orden cognitivo, ejemplos que, por el mismo razonamiento, consideraríamos inconscientes. Estoy apuntando una relación entre causas estilísticas y variación consciente, y causas cognitivas y variación inconsciente que me parece plausible pero que no me atrevo a dar por cierta hasta poder analizar un número mayor y más diversificado de ejemplos.

iii) otros factores a considerar

Algunos comentarios de las entrevistas me han sugerido la posible existencia de otras causas que habría que considerar; son aspectos de carácter semántico y pragmático, aunque también textual:

- según MLC, autora del texto 2, algunas de las variaciones entorno al concepto de "fraseología especializada" son debidas al hecho de tratarse de un concepto que está intentando fijar, que todavía no se encuentra desarrollado en la bibliografía; esta situación de novedad conceptual comporta una indefinición que se refleja en la variación denominativa ${ }^{6}$;

- la misma autora justifica la gran variedad de denominaciones alternativas para "aplicaciones terminográficas" (denominaciones que en la mayoría de casos se refieren a conceptos más concretos) diciendo que por el hecho de que los "vocabularios" son la "aplicación terminográfica" paradigmática, ha recurrido a la denominación de un concepto específico para designar el concepto genérico;

- en el texto 3 original, JML hizo uso de la denominación "grupo especializado" (y otras formas relacionadas, como "grupos de especialistas" y "grupos sociales especializados") en varias ocasiones y, en cambio, no lo hizo durante la entrevista, lo cual provocó un panorama denominativo bastante distinto en el texto nuevo. El autor justifica este hecho explicando que en la parte inicial del artículo (parte que no se trató para este trabajo y que, por 
tanto, el autor no tenía ante los ojos) desarrollaba la noción de "especialidad", noción que retomaba con estas denominaciones para recordar que de esa noción se ha pasado a la de "grupo profesional". En este caso, pues, podríamos hablar de un factor semántico-cognitivo de base textual.

\section{iv) respuestas directas a preguntas directas}

Quisiera terminar la presentación de resultados de la prueba piloto con algunos comentarios generales sobre la variación denominativa verbalizados por los especialistas al final de las entrevistas. Estos comentarios son las respuestas a mis preguntas sobre si varían más de lo que creían, sobre por qué varían en realidad, y sobre si la variación afecta negativamente la eficacia comunicativa. La sensación final es que los especialistas varían más de lo que pensaban, que variar es necesario y que la forma concreta de resolver las necesidades de variación (tanto desde un punto de vista cualitativo como cuantitativo) depende en gran medida del estilo del que escribe y, en definitiva, de su personalidad.

Fragmento de la primera entrevista (con REB):

“Y por qué no has puesto la forma x todo el rato sin parar? Por que no soy sistemática, no me gusta leer textos que empiezan con un patrón y lo van repitiendo sin cesar, me parece cansado, así variado lo encuentro más rico, pero esto tiene que ver con tu forma de escribir y de ser, porque si yo veo algo repetido no me gusta."

Fragmento de la tercera entrevista (con JML):

“¿Varías más de lo que pensabas? Sí, sí [y justifica su variación];

¿No piensas que resulta pesado que en dos páginas hayas utilizado veinte veces grupo y grupo profesional’? Sí, pero no lo sé evitar.

¿No sabes evitarlo o piensas que no hay que evitarlo? No creo que haya que evitarlo en un texto que quiere ser preciso, pero seguro que ahora lo reharía...

¿Y qué harías? [se ríe] ¡Volver a empezar!”

\section{Conclusiones}

Definitivamente, la prueba ha resultado muy positiva y estimulante; en realidad, me ha sorprendido descubrir las posibilidades que abre un análisis de este tipo. No obstante, algunos aspectos metodológicos deberán ser mejorados y algunos sesgos no previstos deberán ser tenidos en cuenta: por ejemplo, al rellenar los vacíos en el texto, el especialista realiza un ejercicio bastante diferente que cuando redacta el texto producido por lo que he llamado "efecto fill in the gaps".

Por otro lado, el análisis realizado ha demostrado que las variables del grupo social deberían ser controladas por cuanto pueden ser relevantes para la interpretación de los resultados; difícilmente puede controlarse la variable "tipo de personalidad", que ha resultado ser pertinente según apuntan los entrevistados, pero sí pueden controlarse aspectos como el sexo y grupo de edad.

Con la prueba piloto pretendía comprobar si la entrevista directa con los especialistas podía aportar nuevos matices a las causas previstas para la variación terminológica; los resultados obtenidos en la prueba me permiten afirmar que no solamente pueden aflorar nuevos matices de causas ya previstas, sino directamente nuevas causas.

Habrá que ahondar en el estudio del grado de consciencia de la variación denominativa; si bien el análisis de coincidencias y divergencias en las denominaciones supone un buen primer paso en ese sentido, hay que prever otras posibilidades más determinantes (o al menos complementarias) para aislar resultados.

En cualquier caso, la prueba demuestra que el ideal de la biunivocidad entre denominación y noción pasa a un segundo nivel de prioridad en la redacción de un texto especializado; el deseo (consciente) de crear un texto estilísticamente "bueno" parece que, finalmente, está más relacionado 
con la eficacia comunicativa que la idealizada biunivocidad, y quizás las necesidades cognitivas (inconscientes), también.

Para terminar, quisiera retomar mis palabras iniciales: "Uno de los problemas terminológicos a que se enfrenta el traductor en la traducción especializada es la variación de los términos (...). Para una buena traducción de la variación en terminología es necesario conocer las causas de la necesidad de variar y los tipos de variación resultantes". Porque el traductor de textos científicotécnicos conoce bien el mencionado ideal de biunivocidad, y para trasladar el fenómeno de la variación terminológica de una lengua a otra es fundamental que conozca también las necesidades estilísticas y cognitivas de los productores de este tipo de textos.

\section{NOTES}

1. De hecho, así mismo se manifestó en ese trabajo anterior, y se presentó la limitación metodológica como una de las vías de trabajo posterior: "Des d'un punt de vista cognitiu, caldria emprendre treballs que permetessin superar la mancança que representa analitzar només el final del procés de producció i formular hipòtesis sobre les altres fases del procés. La realització d'enquestes als productors de textos especialitzats permetria, per exemple, avaluar les causes de la variació des del punt de vista de la producció" (Freixa, 2002:371).

2. Texto 1 (T1): Fragmento inicial de "Elementos lingüísticos de las unidades terminológicas para su extracción automática" (artículo escrito por REB y publicado en La terminología científico-técnica, 2001); Texto 2 (T2):

Fragmento inicial de "Terminología y fraseología especializada: del léxico a la sintaxis" (artículo escrito por MLC y publicado en Panorama actual de la terminología, 2002); Texto 3 (T3): Fragmento de "Terminología, discurso especializado y objetivos sociales" (artículo en prensa escrito por JML).

3. Aún así, en trabajos posteriores me propongo analizar también textos que presenten muy poca variación, y entrevistar a los especialistas sobre las causas de la no variación.

4. Para una discusión acerca de la frontera entre "denominación” y "designación” ver Freixa, 2002:89-92.

5. El concepto "índice de denominación” que aquí presento es una variante del concepto "índice de denominación” desarrollado con anterioridad (Freixa, 2002:224-226).

6. La idea de que la variación conceptual puede ser una causa de la variación denominativa ya la he defendido anteriormente (Freixa, 2002:212).

\section{RÉFÉRENCES}

CABRÉ, M. T. (1999): La terminología: Representación y comunicación. Una teoría de base comunicativa y otros artículos, Sèrie Monographies 3, Barcelona, Universitat Pompeu Fabra.

Ciapuscio, G. (1999): "Variación conceptual y grado de especialidad de los textos", Revista Argentina de Terminología 15.

DIKI-KIDIRI, M. (2000): “Une approche culturelle de la terminologie", Terminologies Nouvelles 21, Terminologie et diversité culturelle, p. 27-31.

FREIXA, J. (2002): La variació terminològica: anàlisi de la variació denominativa en textos de diferent grau d'especialització de l'àrea de medi ambient, Sèrie Tesis 3, Barcelona, Universitat Pompeu Fabra.

GAUDIN, F. (2003): Socioterminologie. Une approche sociolinguistique de la terminologie, Bruxelles, Duculot. KLEIBER, G. (1984): “Dénominations et relations dénominatives”, Langages 76, p. 77-94.

LARA, L.-F. (1999): “Término y cultura: hacia una teoría del término”, in CABRÉ, M. T. (ed.): Terminología y modelos culturales, Barcelona, Universitat Pompeu Fabra, p. 39-60.

SAGER, J.-C. (2000): "In Search of a Foundation: Towards a Theory of the Term", Terminology 5-1, p. 41-58. Temmerman, R. (2001): "Sociocognitive Terminology Theory", in CABRÉ, M. T. and J. FeliU (eds.):

Terminología y cognición. Actas del II Simposio Internacional de Verano de Terminología. (13-16 de julio de 1999), Barcelona, Universitat Pompeu Fabra, p. 75-92. 